\title{
$L(2,1)$-Labeling of the Brick Product Graphs
}

\author{
Xiujun Zhang1,2, Hong Yang2, Hong Li² \\ ${ }^{1}$ School of Information Science and Engineering, Chengdu University, Chengdu, China \\ ${ }^{2}$ Key Laboratory of Pattern Recognition and Intelligent Information Processing, Institutions of Higher Education of Sichuan \\ Province, Chengdu University, Chengdu, China \\ Email: woodszhang@cdu.edu.cn
}

How to cite this paper: Zhang, X.J., Yang, H. and Li, H. (2017) L(2,1)-Labeling of the Brick Product Graphs. Journal of Applied Mathematics and Physics, 5, 1529-1536. https://doi.org/10.4236/jamp.2017.58126

Received: July 15, 2017

Accepted: August 19, 2017

Published: August 23, 2017

Copyright $\odot 2017$ by authors and Scientific Research Publishing Inc. This work is licensed under the Creative Commons Attribution International License (CC BY 4.0).

http://creativecommons.org/licenses/by/4.0/

\begin{abstract}
A $k$ - $L(2,1)$-labeling for a graph $G$ is a function $f: V(G) \rightarrow\{0,1, \cdots, k\}$ such that $|f(u)-f(v)| \geq 2$ whenever $u v \in E(G)$ and $|f(u)-f(v)| \geq 1$ whenever $u$ and $v$ are at distance two apart. The $\lambda$-number for $G$, denoted by $\lambda(G)$, is the minimum $k$ over all $k-L(2,1)$-labelings of $G$. In this paper, we show that $\operatorname{Br}(2 \ell, m, r) \leq 6$ for $\ell=9$ or 11 , which confirms Conjecture 6.1 stated in [X. Li, V. Mak-Hau, S. Zhou, The $L(2,1)$-labelling problem for cubic Cayley graphs on dihedral groups, J. Comb. Optim. (2013) 25: 716-736] in the case when $\ell=9$ or 11 . Moreover, we show that $\operatorname{Br}(2 \ell, m, r)=5$ if 1$)$ either $\ell \equiv 0(\bmod 6), m$ is odd,$r=3$, or 2$) \quad \ell \equiv 0(\bmod 3), m$ is even $(\bmod 2)$, $r=0$.
\end{abstract}

\section{Keywords}

Graph Labeling, Brick Product Graph, L(2,1)-Labeling, Frequency

Assignment Problem

\section{Introduction}

Let $G=(V, E)$ be a graph. For two vertices $u$ and $v$ in $G$, the distance between $u$ and $v$ is the number of the edges of the shortest path between $u$ and $v$. A $k$ - $L(2,1)$-labeling for a graph $G$ is a function $f: V(G) \rightarrow\{0,1, \cdots, k\}$ such that $|f(u)-f(v)| \geq 2$ whenever $u v \in E(G)$ and $|f(u)-f(v)| \geq 1$ whenever $u$ and $v$ are at distance two apart. The $\lambda$-number for $G$, denoted by $\lambda(G)$, is the minimum $k$ over all $k$ - $L(2,1)$-labelings of $G$. This labeling problem of graphs was proposed by Griggs and Roberts [1] which is a variation of the frequency assignment problem introduced by Hale [2]. The frequency assignment problem asks for assigning frequencies to transmitters in a broadcasting network with the aim of avoiding undesired interference. One of the graph theoretical models of the frequency assignment problem is the notion of distance constrained labeling 
of graphs [3] [4] [5].

The $L(2,1)$-labeling problem was studied very extensively in the literature and has attracted much attention. Griggs and Yeh [6] proposed a conjecture, which is called the $\Delta^{2}$-conjecture, that $\lambda(G) \leq \Delta^{2}$ for any graph with $\Delta \geq 2$, where $\Delta$ is the maximum degree of $G$, and they also proved that $\lambda(G) \leq \Delta^{2}+2 \Delta$. Later, it was shown that $\lambda(G) \leq \Delta^{2}+\Delta$ by Chang and Kuo [7], $\lambda(G) \leq \Delta^{2}+\Delta-1$ by Král' and Škrekovski [8], and then $\lambda(G) \leq \Delta^{2}+\Delta-2$ by Goncalves [9]. Until now, this conjecture is still open. Nevertheless, it is still interesting to study the $\Delta^{2}$-conjecture, which has been confirmed for several classes of graphs, such as chordal graphs, outerplanar graphs, generalized Petersen graphs, Hamiltonian graphs with $\Delta \leq 3$, two families of Hamming graphs etc (see [10]). Havet et al. obtained a result implying that the $\Delta^{2}$-conjecture is true for graphs with sufficiently large $\Delta$. Thus, we may need to study the $L(2,1)$-labelling problem for graphs with small $\Delta$. Motivated with this, the $L(2,1)$-labelling problem for the brick product graphs was studied [10].

Let $\ell \geq 2, m \geq 1$ and $r \geq 0$ be integers such that $m+r$ is even. Let $C_{2 \ell}$ be a cycle of length $2 \ell$. The $(m, r)$-brick-product of $C_{2 \ell}$, denoted by $\operatorname{Br}(2 \ell, m, r)$, is the graph with adjacency defined in two cases. For $m=1, r \geq 3$ must be odd and $\operatorname{Br}(2 \ell, 1, r)$ is obtained from the cycle $C_{2 \ell}=\left(v_{0}, v_{1}, v_{2}, \cdots, v_{2 \ell-1}, v_{0}\right)$ by adding chords joining $v_{2 i}$ and $v_{2 i+r}$ for $i=0,1, \cdots, \ell-1$, where subscripts are taken modulo $2 \ell$. In the general case where $m \geq 2, \operatorname{Br}(2 \ell, m, r)$ is obtained by first taking the vertex-disjoint union of $m$ copies of $C_{2 \ell}$, denoted by

$$
C_{2 \ell}(i)=\left(v_{i, 0}, v_{i, 1}, \cdots, v_{i, 2 \ell-1}, v_{i, 0}\right), i=0,1, \cdots, m-1 .
$$

Next, for each pair $(i, j) \in\{0,1, \cdots, m-2\} \times\{0,1, \cdots, 2 \ell-1\}$ such that $i$ and $j$ have the same parity, an edge is added to join $v_{i, j}$ and $v_{i+1, j}$. Finally, for odd $j=1,3, \cdots, 2 \ell-1$, an edge is added to join $v_{0, j}$ and $v_{m-1, j+r}$, where the second subscript is modulo $2 \ell$.

Li et al. [10] proposed the following conjecture:

Conjecture 1. [10] $\lambda(\operatorname{Br}(2 \ell, m, r))=5$ or 6 for all brick products $\operatorname{Br}(2 \ell, m, r)$ with $m \geq 2$ and $m+r \equiv 0(\bmod 2 \ell)$

Shao et al. [11] confirmed the above conjecture, i.e. it was proved that

Theorem 1. [11] $\lambda(\operatorname{Br}(2 \ell, m, r)) \leq 6$ if 1$) \quad \ell$ is even, or 2$) \ell \geq 5$ is odd and $0 \leq r \leq 8$.

Therefore, Conjecture 1 is still open for odd $\ell$ and $r>8$.

In this paper, we show that $\operatorname{Br}(2 \ell, m, r) \leq 6$ for $\ell=9$ or 11 , which confirms Conjecture 6.1 stated in [X. Li, V. Mak-Hau, S. Zhou, The $L(2,1)$-labelling problem for cubic Cayley graphs on dihedral groups, J. Comb. Optim. (2013) 25: 716-736] in the case when $\ell=9$ or 11 . Moreover, we show that $\operatorname{Br}(2 \ell, m, r)=5$ if 1$)$ either $\ell \equiv 0(\bmod 6), m$ is odd $, r=3,2)$ or $\ell \equiv 0(\bmod 3), m$ is even $(\bmod 2), r=0$.

\section{Main Results}

From the definition of the brick product graph, it is clear that 
Fact 1. $\operatorname{Br}(2 \ell, m, r)$ is isomorphic to $\operatorname{Br}(2 \ell, m, 2 \ell-r)$.

\subsection{Some Results on the Upper Bound 6 of $\lambda$-Number}

In [6], it was shown that

Lemma 1. [6] The $\lambda$-number of any connected cubic graph is at least 5 .

Proposition 1. Let $\ell=9$. Then $\lambda(B r(2 \ell, m, r)) \leq 6$ for all $m \geq 3$.

By Theorem 1, we have $\lambda(B r(2 \ell, m, r)) \leq 6$ for all $m \geq 3$ and $r \leq 8$. Together with Fact 1 , we only need to consider $r=9$. Let

$$
P_{3}=\left[\begin{array}{lll}
1 & 3 & 2 \\
4 & 0 & 4 \\
2 & 6 & 6 \\
0 & 4 & 0 \\
6 & 1 & 2 \\
4 & 3 & 5 \\
2 & 0 & 0 \\
6 & 6 & 4 \\
1 & 3 & 2 \\
5 & 5 & 0 \\
0 & 2 & 6 \\
3 & 4 & 1 \\
1 & 6 & 3 \\
4 & 2 & 5 \\
6 & 0 & 0 \\
2 & 5 & 2 \\
4 & 1 & 4 \\
6 & 6 & 0
\end{array}\right],\left[\begin{array}{lllll}
4 & 6 & 4 & 6 & 2 \\
2 & 2 & 0 & 3 & 5 \\
0 & 4 & 5 & 1 & 0 \\
6 & 6 & 2 & 6 & 2 \\
4 & 1 & 0 & 4 & 5 \\
2 & 5 & 3 & 2 & 0 \\
6 & 0 & 1 & 6 & 6 \\
3 & 3 & 5 & 4 & 2 \\
5 & 1 & 2 & 0 & 5 \\
0 & 6 & 4 & 3 & 1 \\
4 & 2 & 1 & 5 & 6 \\
6 & 0 & 6 & 0 & 4 \\
1 & 5 & 4 & 2 & 1 \\
3 & 3 & 1 & 5 & 3 \\
0 & 6 & 6 & 0 & 0 \\
4 & 1 & 3 & 4 & 6 \\
2 & 5 & 5 & 2 & 1 \\
0 & 3 & 1 & 0 & 4
\end{array}\right], P_{7}=\left[\begin{array}{lllllll}
2 & 4 & 2 & 4 & 3 & 1 & 0 \\
0 & 6 & 0 & 6 & 0 & 6 & 2 \\
5 & 2 & 5 & 2 & 5 & 3 & 4 \\
3 & 4 & 1 & 4 & 1 & 0 & 6 \\
1 & 6 & 3 & 6 & 6 & 2 & 3 \\
5 & 2 & 5 & 2 & 0 & 4 & 0 \\
0 & 4 & 0 & 4 & 5 & 1 & 5 \\
6 & 6 & 3 & 6 & 3 & 6 & 3 \\
2 & 0 & 5 & 2 & 1 & 4 & 1 \\
5 & 4 & 1 & 0 & 6 & 2 & 5 \\
3 & 6 & 6 & 3 & 3 & 0 & 3 \\
0 & 1 & 4 & 1 & 5 & 4 & 1 \\
6 & 3 & 0 & 6 & 0 & 2 & 6 \\
1 & 5 & 2 & 2 & 4 & 5 & 0 \\
4 & 0 & 6 & 0 & 6 & 3 & 2 \\
2 & 2 & 4 & 3 & 1 & 0 & 4 \\
0 & 5 & 0 & 5 & 4 & 2 & 1 \\
6 & 1 & 6 & 1 & 6 & 5 & 3
\end{array}\right] .
$$

We use the pattern $P_{m}$ to label $\operatorname{Br}(18, m, 9)$ for $m \in\{3,5,7\}$, and $P_{m}$ induces a 6- $L(2,1)$-labeling of $\operatorname{Br}(18, m, 9)$. Therefore, the case $m<9$ is settled.

$$
Q_{9}=\left[\begin{array}{llll|lllll}
1 & 6 & 4 & 6 & 0 & 2 & 6 & 2 & 1 \\
5 & 3 & 1 & 3 & 5 & 4 & 1 & 0 & 3 \\
2 & 0 & 5 & 0 & 1 & 6 & 3 & 6 & 6 \\
4 & 6 & 2 & 6 & 3 & 2 & 0 & 1 & 4 \\
1 & 3 & 4 & 1 & 0 & 4 & 5 & 3 & 2 \\
6 & 0 & 6 & 3 & 6 & 6 & 1 & 0 & 5 \\
4 & 2 & 1 & 5 & 4 & 2 & 4 & 2 & 3 \\
0 & 5 & 3 & 2 & 0 & 5 & 0 & 5 & 0 \\
3 & 1 & 6 & 4 & 6 & 1 & 6 & 3 & 2 \\
5 & 4 & 2 & 1 & 3 & 4 & 2 & 1 & 6 \\
0 & 6 & 0 & 6 & 0 & 6 & 0 & 4 & 3 \\
2 & 1 & 5 & 4 & 2 & 3 & 5 & 2 & 0 \\
5 & 3 & 3 & 1 & 5 & 1 & 1 & 6 & 6 \\
0 & 0 & 6 & 6 & 0 & 6 & 4 & 4 & 2 \\
4 & 2 & 4 & 2 & 4 & 2 & 2 & 0 & 0 \\
1 & 5 & 1 & 5 & 1 & 0 & 6 & 6 & 4 \\
6 & 3 & 6 & 0 & 6 & 3 & 3 & 1 & 2 \\
4 & 0 & 2 & 2 & 4 & 5 & 0 & 4 & 6
\end{array}\right], \quad\left[\begin{array}{llll|lllllll}
6 & 1 & 4 & 6 & 0 & 6 & 6 & 4 & 5 & 1 & 2 \\
4 & 5 & 2 & 1 & 4 & 3 & 1 & 1 & 3 & 6 & 0 \\
0 & 3 & 0 & 3 & 6 & 0 & 4 & 6 & 0 & 2 & 5 \\
2 & 1 & 4 & 5 & 2 & 5 & 2 & 2 & 5 & 4 & 1 \\
4 & 6 & 6 & 1 & 4 & 1 & 6 & 4 & 1 & 6 & 3 \\
0 & 3 & 0 & 3 & 6 & 3 & 0 & 0 & 3 & 2 & 5 \\
5 & 1 & 2 & 5 & 0 & 5 & 5 & 2 & 5 & 0 & 0 \\
3 & 6 & 4 & 1 & 3 & 1 & 3 & 4 & 1 & 4 & 6 \\
0 & 2 & 0 & 6 & 6 & 4 & 6 & 0 & 6 & 2 & 3 \\
4 & 5 & 3 & 2 & 0 & 0 & 2 & 5 & 3 & 0 & 5 \\
6 & 0 & 1 & 5 & 3 & 5 & 4 & 1 & 1 & 6 & 2 \\
2 & 3 & 6 & 0 & 6 & 1 & 6 & 6 & 4 & 3 & 0 \\
4 & 1 & 2 & 4 & 2 & 4 & 3 & 0 & 0 & 5 & 6 \\
0 & 6 & 0 & 6 & 0 & 0 & 5 & 4 & 2 & 1 & 4 \\
5 & 3 & 5 & 3 & 4 & 2 & 1 & 6 & 5 & 3 & 2 \\
2 & 0 & 2 & 0 & 6 & 6 & 3 & 3 & 1 & 0 & 5 \\
4 & 6 & 6 & 4 & 3 & 1 & 0 & 5 & 6 & 2 & 1 \\
0 & 3 & 0 & 2 & 5 & 4 & 2 & 2 & 0 & 4 & 6
\end{array}\right] .
$$


Now, we consider the case $m \geq 9$. If $m=4 k+5$ for $k \geq 1$, we obtain a 6$L(2,1)$-labeling of $\operatorname{Br}(18, m, 9)$ by repeating the leftmost four columns of $Q_{9}$; If $m=4 k+7$ for $k \geq 1$, we obtain a $6-L(2,1)$-labeling of $\operatorname{Br}(18, m, 9)$ by repeating the leftmost four columns of $Q_{11}$ (see Figure 1). Therefore, $\lambda(B r(2 \ell, m, r)) \leq 6$ for $\ell=9$ and $m \geq 3$.

Proposition 2. Let $\ell=11$. Then $\lambda(B r(2 \ell, m, r)) \leq 6$ for all $m \geq 3$.

Similar to Proposition 1, we only need to consider the case $r=9$ and 11 .

Case 1: $r=9$.

We use the following pattern $P_{m}$ to label $\operatorname{Br}(22, m, 9)$ for $m \in\{3,5\}$, and $P_{m}$ induces a $6-L(2,1)$-labeling of $\operatorname{Br}(22, m, 9)$. Therefore, the case $m \leq 5$ is settled. Now, we consider the case $m \geq 7$. If $m=4 k+3$ for $k \geq 1$, we obtain a 6- $L(2,1)$-labeling of $\operatorname{Br}(22, m, 9)$ by repeating the leftmost four columns of $Q_{7}$; If $m=4 k+5$ for $k \geq 1$, we obtain a 6 - $L(2,1)$-labeling of $\operatorname{Br}(22, m, 9)$ by repeating the leftmost four columns of $Q_{9}$. Therefore, $\lambda(\operatorname{Br}(2 \ell, m, r)) \leq 6$ for $\ell=11$ and $m \geq 3$.

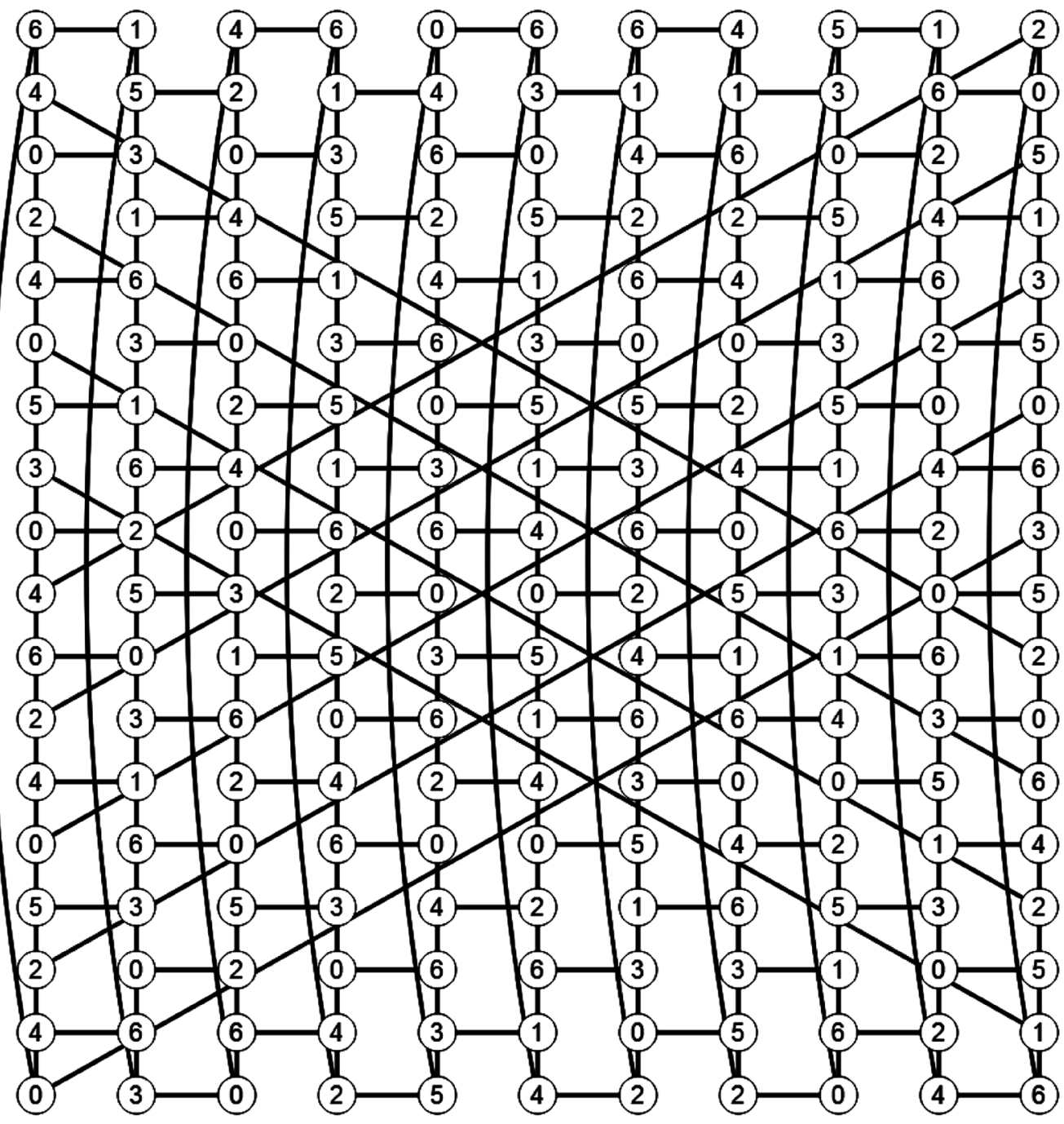

Figure 1. The 6- $L(2,1)$-labeling of $\operatorname{Br}(18,11,9)$ induced by $Q_{11}$. 


\begin{tabular}{|c|c|c|c|c|c|c|c|c|c|c|c|c|c|c|c|c|c|c|c|c|c|c|c|c|c|c|c|}
\hline & 3 & 0 & 07 & & 4 & 1 & 1 & 3 & $1]$ & & {$[6$} & 0 & 0 & 6 & 6 & 1 & $2]$ & & 2 & 6 & 3 & 1 & 2 & 6 & 4 & 6 & 6 \\
\hline & 1 & 1 & 3 & & 2 & 0 & 4 & 2 & 4 & & 2 & 4 & 2 & 4 & 0 & 3 & 0 & & 4 & 4 & 0 & 6 & 4 & 0 & 2 & 0 & 4 \\
\hline & 0 & 4 & 4 & & 1 & 3 & 3 & 0 & 0 & & 5 & 1 & 5 & 1 & 2 & 5 & 6 & & 0 & 2 & 5 & 2 & 1 & 3 & 5 & 3 & 2 \\
\hline & 3 & 3 & 0 & & 4 & 4 & 1 & 1 & 3 & & 0 & 3 & 0 & 6 & 4 & 0 & 4 & & 6 & 6 & 1 & 4 & 6 & 6 & 0 & 6 & 0 \\
\hline & 4 & 1 & 1 & & 3 & 0 & 0 & 4 & 2 & & 2 & 5 & 4 & 2 & 1 & 3 & 2 & & 3 & 0 & 3 & 0 & 0 & 2 & 2 & 4 & 3 \\
\hline & 2 & 0 & 4 & & 1 & 1 & 3 & 3 & 0 & & 6 & 0 & 6 & 0 & 6 & 6 & 0 & & 5 & 2 & 5 & 2 & 5 & 4 & 6 & 1 & 6 \\
\hline & 1 & 3 & 3 & & 2 & 4 & 4 & 1 & 4 & & 1 & 4 & 1 & 4 & 4 & 2 & 4 & & 0 & 6 & 0 & 6 & 3 & 1 & 0 & 3 & 0 \\
\hline & 4 & 4 & 1 & & 0 & 0 & 2 & 0 & 2 & & 5 & 2 & 5 & 2 & 0 & 0 & 6 & & 2 & 1 & 4 & 4 & 0 & 6 & 2 & 6 & 2 \\
\hline & 3 & 0 & 0 & & 1 & 3 & 1 & 3 & 1 & & 3 & 0 & 0 & 6 & 3 & 5 & 1 & & 6 & 3 & 6 & 1 & 2 & 4 & 5 & 0 & 4 \\
\hline & 1 & 1 & 3 & & 4 & 2 & 4 & 2 & 4 & & 1 & 6 & 3 & 4 & 1 & 2 & 4 & & 0 & 5 & 0 & 3 & 5 & 0 & 3 & 2 & 6 \\
\hline & 0 & 4 & 4 & & 3 & 0 & 3 & 0 & 0 & & 5 & 2 & 5 & 2 & 5 & 0 & 0 & & 4 & 1 & 2 & 6 & 1 & 6 & 6 & 4 & 1 \\
\hline & 3 & 3 & 0 & $P_{5}=$ & 1 & 4 & 1 & 1 & 3 & & 3 & 4 & 0 & 0 & 3 & 3 & 6 & & 2 & 6 & 4 & 0 & 4 & 4 & 2 & 0 & 5 \\
\hline & 4 & 1 & 1 & & 0 & 2 & 2 & 4 & 2 & & 6 & 1 & 2 & 6 & 6 & 1 & 4 & & 5 & 3 & 1 & 5 & 2 & 0 & 5 & 3 & 2 \\
\hline & 2 & 2 & 4 & & 3 & 3 & 0 & 3 & 0 & & 4 & 3 & 5 & 1 & 4 & 5 & 2 & & 1 & 0 & 6 & 3 & 6 & 6 & 1 & 6 & 4 \\
\hline & 3 & 0 & 0 & & 4 & 1 & 4 & 1 & 4 & & 0 & 6 & 0 & 3 & 0 & 3 & 0 & & 4 & 2 & 4 & 0 & 0 & 2 & 4 & 2 & 1 \\
\hline & 1 & 1 & 3 & & 2 & 0 & 2 & 0 & 2 & & 2 & 2 & 4 & 5 & 2 & 6 & 4 & & 6 & 5 & 1 & 2 & 5 & 5 & 0 & 0 & 3 \\
\hline & 0 & 4 & 2 & & 1 & 3 & 1 & 3 & 3 & & 4 & 0 & 6 & 0 & 4 & 1 & 2 & & 1 & 3 & 6 & 4 & 1 & 3 & 3 & 6 & 5 \\
\hline & 3 & 3 & 0 & & 4 & 2 & 4 & 4 & 1 & & 6 & 5 & 2 & 3 & 6 & 3 & 0 & & 5 & 0 & 2 & 0 & 6 & 6 & 1 & 4 & 1 \\
\hline & 4 & 1 & 4 & & 3 & 0 & 0 & 2 & 2 & & 0 & 3 & 4 & 1 & 2 & 5 & 6 & & 2 & 6 & 5 & 3 & 2 & 0 & 5 & 0 & 3 \\
\hline & 0 & 0 & 2 & & 1 & 1 & 3 & 3 & 0 & & 2 & 6 & 0 & 6 & 0 & 0 & 4 & & 4 & 4 & 0 & 1 & 4 & 4 & 2 & 2 & 6 \\
\hline & 1 & 3 & 1 & & 0 & 4 & 4 & 1 & 4 & & 5 & 1 & 2 & 4 & 5 & 2 & 1 & & 6 & 2 & 2 & 6 & 6 & 1 & 6 & 4 & 0 \\
\hline & 4 & 2 & 4 & & 3 & 3 & 0 & 0 & 2 & & 3 & 3 & 5 & 1 & 3 & 4 & 6 & & 0 & 0 & 5 & 4 & 0 & 3 & 0 & 1 & 3 \\
\hline & 6 & 2 & 27 & & 4 & 0 & 0 & 2 & 37 & & 5 & 3 & 2 & 6 & 2 & 6 & 4 & & 5 & 3 & 4 & 1 & 4 & 2 & 1 & 6 & 3 \\
\hline & 3 & 0 & 4 & & 6 & 2 & 5 & 4 & 0 & & 2 & 0 & 5 & 0 & 5 & 0 & 2 & & 0 & 6 & 0 & 3 & 6 & 0 & 4 & 4 & 1 \\
\hline & 1 & 5 & 6 & & 0 & 4 & 3 & 6 & 5 & & 4 & 6 & 1 & 3 & 1 & 3 & 6 & & 4 & 2 & 2 & 5 & 1 & 5 & 2 & 0 & 5 \\
\hline & 4 & 3 & 1 & & 5 & 6 & 0 & 1 & 3 & & 0 & 2 & 4 & 6 & 4 & 5 & 0 & & 6 & 0 & 4 & 0 & 3 & 3 & 6 & 6 & 2 \\
\hline & 0 & 6 & 4 & & 3 & 1 & 2 & 4 & 0 & & 3 & 5 & 0 & 2 & 0 & 2 & 3 & & 3 & 5 & 6 & 2 & 5 & 1 & 4 & 1 & 4 \\
\hline & 3 & 2 & 0 & & 0 & 4 & 6 & 6 & 2 & & 1 & 1 & 6 & 4 & 6 & 4 & 1 & & 0 & 1 & 3 & 4 & 0 & 6 & 0 & 3 & 0 \\
\hline & 1 & 5 & 5 & & 6 & 2 & 1 & 3 & 4 & & 5 & 3 & 3 & 0 & 3 & 0 & 6 & & 2 & 4 & 5 & 1 & 2 & 4 & 2 & 5 & 2 \\
\hline & 6 & 3 & 1 & & 3 & 0 & 4 & 5 & 1 & & 2 & 0 & 5 & 6 & 1 & 2 & 4 & & 5 & 6 & 0 & 3 & 5 & 0 & 6 & 0 & 6 \\
\hline & 2 & 0 & 6 & & 1 & 5 & 6 & 2 & 3 & & 4 & 6 & 1 & 3 & 4 & 6 & 0 & & 1 & 3 & 4 & 6 & 1 & 3 & 1 & 3 & 3 \\
\hline & 4 & 5 & 2 & & 6 & 3 & 1 & 4 & 6 & & 1 & 2 & 4 & 5 & 2 & 1 & 5 & & 4 & 5 & 1 & 0 & 4 & 6 & 4 & 6 & 0 \\
\hline & 0 & 3 & 4 & ${ }^{\prime}=$ & 2 & 0 & 5 & 0 & 0 & $O^{\prime}=$ & 3 & 0 & 6 & 0 & 0 & 4 & 3 & & 2 & 0 & 6 & 3 & 2 & 0 & 0 & 2 & 4 \\
\hline & 6 & 6 & 1 & & 5 & 6 & 2 & 3 & 5 & & 6 & 5 & 3 & 4 & 6 & 2 & 0 & & 5 & 4 & 2 & 1 & 6 & 3 & 6 & 5 & 1 \\
\hline & 1 & 4 & 5 & & 0 & 3 & 4 & 6 & 2 & & 0 & 2 & 0 & 2 & 1 & 5 & 6 & & 0 & 6 & 0 & 4 & 4 & 1 & 4 & 0 & 6 \\
\hline & 3 & 2 & 0 & & 2 & 5 & 0 & 0 & 4 & & 4 & 6 & 4 & 6 & 3 & 3 & 1 & & 3 & 2 & 5 & 6 & 0 & 6 & 2 & 2 & 4 \\
\hline & 0 & 6 & 6 & & 6 & 1 & 2 & 5 & 1 & & 1 & 3 & 2 & 0 & 5 & 0 & 5 & & 1 & 4 & 3 & 1 & 2 & 4 & 0 & 6 & 0 \\
\hline & 2 & 4 & 2 & & 4 & 3 & 6 & 3 & 6 & & 6 & 0 & 5 & 4 & 2 & 4 & 2 & & 6 & 6 & 0 & 4 & 6 & 1 & 3 & 4 & 2 \\
\hline & 6 & 0 & 5 & & 1 & 5 & 4 & 0 & 4 & & 2 & 4 & 3 & 1 & 0 & 6 & 6 & & 0 & 2 & 5 & 2 & 0 & 5 & 6 & 0 & 6 \\
\hline & 3 & 3 & 1 & & 6 & 0 & 2 & 6 & 1 & & 0 & 6 & 0 & 6 & 3 & 2 & 4 & & 4 & 4 & 1 & 6 & 4 & 2 & 4 & 2 & 4 \\
\hline & 1 & 5 & 4 & & 2 & 4 & 5 & 3 & 5 & & 5 & 1 & 2 & 4 & 5 & 0 & 0 & & 1 & 6 & 3 & 0 & 1 & 6 & 0 & 6 & 0 \\
\hline & 4 & 0 & 2 & & 0 & 6 & 0 & 0 & 2 & & 2 & 3 & 6 & 0 & 2 & 6 & 3 & & 5 & 2 & 5 & 2 & 5 & 4 & 2 & 1 & 3 \\
\hline & 2 & 6 & 6 & & 5 & 1 & 2 & 4 & 4 & & 4 & 0 & 1 & 5 & 4 & 1 & 5 & & 0 & 4 & 0 & 4 & 3 & 1 & 6 & 4 & 6 \\
\hline & 0 & 4 & 0 & & 2 & 3 & 6 & 6 & $1]$ & & 1 & 6 & 4 & 3 & 0 & 3 & 0 & & 2 & 1 & 6 & 6 & 0 & 5 & 3 & 2 & 0 \\
\hline
\end{tabular}

Case 2: $r=11$.

We use the following pattern $P_{m}^{\prime}$ to label $\operatorname{Br}(22, m, 11)$ for $m \in\{3,5\}$, and $P_{m}^{\prime}$ induces a 6- $L(2,1)$-labeling of $\operatorname{Br}(22, m, 11)$. Therefore, the case $m \leq 5$ is settled. Now, we consider the case $m \geq 7$. If $m=4 k+3$ for $k \geq 1$, we obtain a 
6- $L(2,1)$-labeling of $\operatorname{Br}(22, m, 11)$ by repeating the leftmost four columns of $Q_{7}^{\prime}$; If $m=4 k+5$ for $k \geq 1$, we obtain a 6- $L(2,1)$-labeling of $\operatorname{Br}(22, m, 11)$ by repeating the leftmost four columns of $Q_{9}^{\prime}$. Therefore, $\lambda(\operatorname{Br}(2 \ell, m, r)) \leq 6$ for $\ell=11$ and $m \geq 3$.

From Propositions 1 and 2, we have

Theorem 2. Let $m \geq 3$. Then we have $\lambda(B r(2 \ell, m, r)) \leq 6$ for $\ell=9$ or 11 .

\subsection{Brick Product Graphs with $\lambda$-Number 5}

In [10], it was proved that

Theorem 3. Let $\ell, m \geq 2$ and $r \geq 0$ be integers such that $m+r \equiv 0(\bmod 2 \ell)$. Then

$$
5 \leq \lambda(B r(2 \ell, m, r)) \leq 7 .
$$

Moreover, $\lambda(\operatorname{Br}(2 \ell, m, r))=5$ if and only if one of the following holds:

1) 3 divides $\ell$ and 6 divides $m$;

2) 6 divides $\ell$ and 3 divides $m$.

Furthermore, if neither 1) nor 2) is satisfied, then $\lambda(\operatorname{Br}(2 \ell, m, r))=6$ provided that $m=2$ (and $\ell$ is even or odd), or both $\ell$ and $m$ are even.

However, Theorem 3 consider the condition that $m+r \equiv 0(\bmod 2 \ell)$. There may exist other brick product graphs with $\lambda$-number 5 with the condition $m+r \equiv 0(\bmod 2 \ell)$. We provide some brick product graphs $\operatorname{Br}(2 \ell, m, r)$ with $\lambda$-number 5 in the following:

Theorem 4. Let $\ell \equiv 0(\bmod 3), m \equiv 0(\bmod 2)$ with $m \geq 4, r=0$. Then $\lambda(\operatorname{Br}(2 \ell, m, r))=5$.

Let $m=2 k, \quad P=\left[\begin{array}{cc}5 & 2 \\ 1 & 4 \\ 3 & 0\end{array}\right], \quad P_{1}=P^{k}=\underbrace{P P \cdots P}_{k \text { times }}$ and $Q=\left[\begin{array}{c}P_{1} \\ P_{1} \\ \vdots \\ P_{1}\end{array}\right]$, where $P_{1}$ is used for $\frac{2 \ell}{3}$ times. Then $Q$ induces a 5- $L(2,1)$-labeling of $\operatorname{Br}(2 \ell, m, r)$, and so $\lambda(B r(2 \ell, m, r)) \leq 5$.

Proposition 3. Let $\ell \equiv 0(\bmod 6), m=3, r=3$. Then $\lambda(B r(2 \ell, m, r))=5$.

Let $P=\left[\begin{array}{lll}2 & 0 & 5 \\ 5 & 4 & 2 \\ 3 & 1 & 0 \\ 0 & 5 & 3 \\ 4 & 2 & 1 \\ 1 & 0 & 4 \\ 5 & 3 & 2 \\ 2 & 1 & 5 \\ 0 & 4 & 3 \\ 3 & 2 & 0 \\ 1 & 5 & 4 \\ 4 & 3 & 1\end{array}\right]$, and $Q=\left[\begin{array}{c}P \\ P \\ \vdots \\ P\end{array}\right]$, where $P$ is used for $\frac{\ell}{3}$ times. Then $Q$ 
induces a 5- $L(2,1)$-labeling of $\operatorname{Br}(2 \ell, m, r)$, and so $\lambda(\operatorname{Br}(2 \ell, m, r)) \leq 5$.

Proposition 4. Let $\ell \equiv 0(\bmod 6), m=5, r=3$. Then $\lambda(B r(2 \ell, m, r))=5$.

Let $P=\left[\begin{array}{lllll}1 & 3 & 4 & 0 & 1 \\ 5 & 0 & 2 & 3 & 5 \\ 2 & 4 & 5 & 1 & 2 \\ 0 & 1 & 3 & 4 & 0 \\ 3 & 5 & 0 & 2 & 3 \\ 1 & 2 & 4 & 5 & 1 \\ 4 & 0 & 1 & 3 & 4 \\ 2 & 3 & 5 & 0 & 2 \\ 5 & 1 & 2 & 4 & 5 \\ 3 & 4 & 0 & 1 & 3 \\ 0 & 2 & 3 & 5 & 0 \\ 4 & 5 & 1 & 2 & 4\end{array}\right]$, and $Q=\left[\begin{array}{c}P \\ P \\ \vdots \\ P\end{array}\right]$, where $P$ is used for $\frac{\ell}{3}$ times.

Then $Q$ induces a 5-L(2,1)-labeling of $\operatorname{Br}(2 \ell, m, r)$, and so $\lambda(B r(2 \ell, m, r)) \leq 5$.

Proposition 5. Let $\ell \equiv 0(\bmod 6), m=7, r=3$. Then $\lambda(B r(2 \ell, m, r))=5$.

Let $P=\left[\begin{array}{ccccccc}1 & 5 & 4 & 2 & 1 & 5 & 4 \\ 4 & 3 & 1 & 0 & 4 & 3 & 1 \\ 2 & 0 & 5 & 3 & 2 & 0 & 5 \\ 5 & 4 & 2 & 1 & 5 & 4 & 2 \\ 3 & 1 & 0 & 4 & 3 & 1 & 0 \\ 0 & 5 & 3 & 2 & 0 & 5 & 3 \\ 4 & 2 & 1 & 5 & 4 & 2 & 1 \\ 1 & 0 & 4 & 3 & 1 & 0 & 4 \\ 5 & 3 & 2 & 0 & 5 & 3 & 2 \\ 2 & 1 & 5 & 4 & 2 & 1 & 5 \\ 0 & 4 & 3 & 1 & 0 & 4 & 3 \\ 3 & 2 & 0 & 5 & 3 & 2 & 0\end{array}\right]$, and $Q=\left[\begin{array}{c}P \\ P \\ \vdots \\ P\end{array}\right]$, where $P$ is used for $\frac{\ell}{3}$

times. Then $Q$ induces a 5-L(2,1)-labeling of $\operatorname{Br}(2 \ell, m, r)$, and so $\lambda(B r(2 \ell, m, r)) \leq 5$.

Proposition 6. Let $\ell \equiv 0(\bmod 6), m=9, r=3$. Then $\lambda(B r(2 \ell, m, r))=5$.

Let $P=\left[\begin{array}{ccccccccc}1 & 5 & 4 & 2 & 1 & 5 & 4 & 2 & 3 \\ 4 & 3 & 1 & 0 & 4 & 3 & 1 & 2 & 0 \\ 2 & 0 & 5 & 3 & 2 & 0 & 5 & 4 & 3 \\ 5 & 4 & 2 & 1 & 5 & 4 & 2 & 1 & 5 \\ 3 & 1 & 0 & 4 & 3 & 1 & 0 & 3 & 2 \\ 0 & 5 & 3 & 2 & 0 & 5 & 3 & 0 & 4 \\ 4 & 2 & 1 & 5 & 4 & 2 & 1 & 2 & 1 \\ 1 & 0 & 4 & 3 & 1 & 0 & 4 & 5 & 3 \\ 5 & 3 & 2 & 0 & 5 & 3 & 2 & 1 & 0 \\ 2 & 1 & 5 & 4 & 2 & 1 & 5 & 4 & 2 \\ 0 & 4 & 3 & 1 & 0 & 4 & 3 & 0 & 5 \\ 3 & 2 & 0 & 5 & 3 & 2 & 0 & 3 & 1\end{array}\right]$, and $Q=\left[\begin{array}{c}P \\ P \\ \vdots \\ P\end{array}\right]$, where $P$ is used for 
$\frac{\ell}{3}$ times. Then $Q$ induces a 5- $L(2,1)$-labeling of $\operatorname{Br}(2 \ell, m, r)$, and so $\lambda(\operatorname{Br}(2 \ell, m, r)) \leq 5$.

By observing the results of Propositions 3 - 6, we propose the following conjecture:

Conjecture 2. Let $\ell \equiv 0(\bmod 6), m \equiv 1(\bmod 2), \quad r=3$. Then $\lambda(B r(2 \ell, m, r))=5$.

\section{Acknowledgements}

This work was supported by Applied Basic Research (Key Project) of Sichuan Province under grant 2017JY0095, Key Project of Sichuan Provincial Department of Education under grant 17ZA0079 and Automotive Creative Design Pilot Area of Chengdu University and Longquanyi District under grant 2015-CX0000010-ZF.

\section{References}

[1] Roberts, F.S. (1988) Private Communication to J. R. Griggs.

[2] Hale, W.K. (1980) Frequency Assignment: Theory and Applications. Annals of Operations Research, 76, 73-93. https://doi.org/10.1109/PROC.1980.11899

[3] Whittlesey, M.A., Georges, J.P. and Mauro, D.W. (1995) On the $\lambda$ number of Qn and related graphs. SIAM Journal on Discrete Mathematics, 8, 499-506. https://doi.org/10.1137/S0895480192242821

[4] Shao, Z. and Vesel, A. (2014) L(2,1) -Labeling of the Strong Product of Paths and Cycles. The Scientific World Journal, 2014, 12.

[5] Georges, J.P., Mauro, D.W. and Whittlesey, M.A. (1994) Relating Path Coverings to Vertex Labellings with a Condition at Distance Two. Discrete Mathematics, 135, 103-111.

[6] Griggs, J.R. and Yeh, R.K. (1992) Labelling Graphs with a Condition at Distance Two. SIAM Journal on Discrete Mathematics, 5, 586-595. https://doi.org/10.1137/0405048

[7] Chang, G.J. and Kuo, D. (1996) The L(2,1) -Labeling Problem on Graphs. SIAM Journal on Discrete Mathematics, 9, 309-316. https://doi.org/10.1137/S0895480193245339

[8] Král', D. and Škrekovski, R. (2003) A Theorem about Channel Assignment Problem. SIAM Journal on Discrete Mathematics, 16, 426-437. https://doi.org/10.1137/S0895480101399449

[9] Goncalves, D. (2008) On the $L(p, 1)$-Labelling of Graphs. Discrete Mathematics, 308, 1405-1414. https://doi.org/10.1016/j.disc.2007.07.075

[10] Li, X., Mak-Hau, V. and Zhou, S. (2013) The L(2,1) -Labelling Problem for Cubic Cayley Graphs on Dihedral Groups. Journal of Combinatorial Optimization, 25, 716-736. https://doi.org/10.1007/s10878-012-9525-4

[11] Shao, Z., Xu, J. and Yeh, R.K. (2016) L(2,1) -Labeling for Brick Product Graphs. Journal of Combinatorial Optimization, 31, 447-462. https://doi.org/10.1007/s10878-014-9763-8 
Submit or recommend next manuscript to SCIRP and we will provide best service for you:

Accepting pre-submission inquiries through Email, Facebook, LinkedIn, Twitter, etc. A wide selection of journals (inclusive of 9 subjects, more than 200 journals)

Providing 24-hour high-quality service

User-friendly online submission system

Fair and swift peer-review system

Efficient typesetting and proofreading procedure

Display of the result of downloads and visits, as well as the number of cited articles Maximum dissemination of your research work

Submit your manuscript at: http://papersubmission.scirp.org/

Or contact jamp@scirp.org 